\title{
Bomarzo: il santuario neoplatonico
}

\section{Elémire Zolla}

Lettere di Annibal Caro del 1564 al Duca Vicino Orsini, signore di Bomarzo, accennano a teatri, stravaganze, meraviglie soprannaturali in quel feudo. Quindi la proprietà del luogo passa ai Della Rovere e ad altri ancora, ma l'esistenza di edifizi e statue senza eguali resta ovvia ed inavvertita per secoli.

Una leggenda rustica del luogo dice che gli Orsini avrebbero messo all'opera turchi prigionieri di Lepanto. Un'altra parla di spose popolane deflorate dai feudatari usi a far sparire quelle che scoprissero peccatrici dentro una botola in comunicazione col bosco. È tanto che si possano raccogliere le leggende, l'oblio stendendosi su tutto; non si sa nemmeno piú dove sieno le reliquie etrusche annunciate dalle vecchie guide come ornamento del municipio insediato nel castello. Appoggiati ad una finestra del castello, si guarda alla valle dove il verde tenero e vaste macchie di un grigio inazzurrato si stendono soavemente, si tenta di dimenticare le chiacchiere di quanti hanno ansiosamente tentato di persuadere che le statue furono divertimenti senza senso, spiritosi e di gusto greve, si segue con l'occhio la linea del viottolo nella valle, si coglie il bianchieggiare del tempio levato fra le statue, in mezzo al boschetto. Occorre cacciare come noiosi fantasmi coloro che hanno contaminato il luogo con la loro bonarietà o la loro eccitazione.

Bomarzo è raccolta attorno al castello, alto sullo sperone di roccia, fra le convalli di tufo. Il luogo rammenta altre rocche già etrusche del Lazio, con selve sonore di cascate dominate da colli ricchi di antri: Vejo, Vejano.

Sul terrazzo del castello sono incise alcune targhe: "Mangia, bevi e gioca. Disprezza le cose della terra. Dupo morte nessuna voluttà. Dopo morte vera voluttà" e, fra le frasi contraddittorie, "I beati tennero la via di mezzo." Oppure, ancora: "Il sapiente sarà dominatore degli astri. La prudenza è minore del fato. E dunque?" "Sapiens dominabitur astris," fu un motto della setta rosacrociana, né la via di mezzo o l'ironia del "Quid ergo?" sono emblemi dello scetticismo di chi scansi il pensiero perché disperante, come vorrebbe una lettura infastidita e frettolosa. Via di mezzo è 
infatti la voluttà di chi sta sulla terra come dopo la morte, che trova felicità nel "conoscersi, vincersi, vivere a sé stesso," come è detto in un'altra scritta. Il terrazzo guarda il declivio che si stende verso occidente, prima coltivato, poi folto d'alberi. Un torrente riga la campagna, si insinua nel bosco, il tempietto e le statue che oggi ne affiorano, dovettero essere celate nel folto.

Se si scende nel bosco si scoprono gli elementi necessari d'ogni santuario: acqua corrente, antri dove il tufo è acclive, alberi; e "aram nemus vult." Un santuario al culto della bizzaria e del rimpianto per la morta Giulia Farnese? Spacciandosi per questo la verità del luogo si nasconde agli sguardi freddi. Ma come non riconoscere le vestigia di un percorso labirintico, dedicato all'antica religione di Porfirio e di Giamblico e della soave Ipazia, riesumata alla tavolata di Lorenzo de' Medici, da Lodovico Lazzarelli, dal Pontano?

I racconti dei villici spauriti sono l'unico riflesso che è consentito a certi culti di proiettare nelle menti materiali e fosche, che associano ogni cosa sottile e inconsueta al proibito, alla violenza, al contatto con lo straniero. Né la Chiesa reprimeva benché il concilio di Trento fosse già celebrato, (anzi il nome del cardinale Mandruzzo che vi partecipò è conservato in uno degli edifici sparsi nel boschetto); nel secolo seguente sarà concessa licenza di stampare opere neoplatoniche ed ermetiche come quella di Della Riviera, e a Roma verrà edificato quel tempietto di cui si conserva ancora una "porta magica" e due statue in un angolo di piazza Vittorio (pressoché celata, su una rovina abitata da gatti dietro uno strepitoso mercato, fra stagni coperti da un velo di tritumi). Il cardinale Mandruzzo durante il concilio di Trento aveva fatto stampare opere cabbalistiche ad Ala ed aveva altresí impedito la loro condanna: ecco un buon avvio a capire Bomarzo.

$\mathrm{Al}$ bordo del bosco dove il terreno comincia a digradare verso il cuore delle valle, si alza il tempietto che accoglie nel santuario.

Il pronao posa su quattro ordini di colonne corinzie e dietro sta una cella ottagonale. Agio, grazia infonde un festone che corre alla base, ma tuttavia le forme angolose, maschili del pronao si fondono in modo precario, teso con la cella a cupola, ottagonale come le fonti battesimali, i luoghi di rinascita.

Dietro comincia la discesa, per una scalinata dominata da un cane a tre fauci, simbolo infernale, che, dice il Fulgentius Metaphoralis sta ai piedi della conoscenza del futuro, alla base della previdenza che ci guarda dalla inferni declinationem. Il cane simboleggia con le tre bocche il morso della fatica (di chi brami acquisti), il morso del timore (che angustia i possidenti), il morso del dolore (che costerà lasciare i beni di fortuna). 
A destra la scalinata di pietra s'apre su una platea rettangolare, delimitata da grosse pigne e ghiande alternate. In fondo alla spianata due orsi reggono rose di peperino.

La previdenza quale oggetto può avere, piú consono dei germi compatti e gravidi? Essa, dice Giamblico nel De Mysteriis (III,I), piglia origine dal divino, cioè dalla causa prima che contiene in sé ciò che poi largisce agli esseri che da essa discendono, "ha l'essenza e la causa di ciò che è, donde proviene l'incessante possesso della preveggenza." Immutevoli e ignei sono gli astri che ruotano nel cielo, e di pari natura sono dunque le specie vegetali e animali che seguono in perfetta obbedienza il loro ciclo di nascita, crescita, morte, rinascita; conoscenza del futuro, non è se non individuazione di questi cicli ordinati, è il prevedere la cosa formata già osservando la sua virtualità di germe. Il germe è simile al fuoco perché tende all'alto, cosí la pigna, segno di fertilità, di abbondante provvidenza. Lasciato alle spalle il Cerbero, lo spirito acquisitivo, ci si apre a questa successione di semi, alla fertilità della mente abbandonata. Si impara a discernere anche il destino iscritto nelle cose, la pianta nel seme, cui potrà contrastare la ventura o caso o fortuna o sorte, ma che resta il segno della volontà divina, del fato e delle stelle cui si deve adeguare con la volontà.

Vasta è la platea fra le ghiande e le pigne, fino ai due orsi che le chiudono, a guardia d'un angolo racchiuso fra arbusti. Gli orsi sono bestie araldiche degli Orsini, ma in sé rappresentano l'Orsa maggiore, il punto d'orientamento nella tenebra e reggono la rosa, il simbolo della voluttà, dell'effimero gioioso e della spontanea prodigalità in virtú del profumo che spande senza risparmio. La rosa significa altresí la saggezza, e infine la segretezza, sub rosa saranno le rivelazioni capitali, in fondo alla platea. Si penetra nell'angolo segreto, che ha sullo sfondo il castello, e sta sopra un avallamento, a sperone. Nel centro due leoni, con un cucciolo al fianco della leonessa. A sinistra una donna dal sereno volto fra le due bande di capelli, dal corpo vezzoso, che alla vita diventa pesce e poggia col pube sulla terra, sdoppiandosi in due scagliose code divaricate. A destra un'altra donna di duplice natura fissa l'oriente, il suo corpo nudo è privo di braccia e di gambe, apre due ali come di pipistrello sopra una coda.

Ursine sono dette, madre dell'orso, matres ursinae o Melusine nel Medio Evo le forze che reggono lo spazio, le potenze liberatrici e ambigue dell'istinto. Nelle leggende medievali esse sposano mortali che compiono l'errore di spiarle il giorno di Saturno, della male sorte, quando tornano alla loro forma anfibia, mostruosa, connessa all'elemento dell'acqua (pinne) e dell'aria (ali). In questo angolo stanno rivelate nella loro forma mostruosa, casuale ri- 
spetto al loro destino di spose di mortali predestinati ad alte imprese, i fondatori di dinastie. I due leoni stesi fra le Melusine sono il simbolo alchemico del corpo e dello spirito; guardano infatti in direzioni opposte, attratti dall'aria e dall'acqua in cui si possono sciogliere se divisi.

Fra queste figure che non guardano chi le contempla, si riposa ascoltando il fruscio del torrente nascosto, poi si cala per un pendío, si segue un sentiero che gira in vista del tempietto, ad arco attorno ad esso, per dilungarsi assai lontano, verso una cascata. Un masso rovesciato sul cammino, rivela, a scostare la terra, altorilievi di figure: un essere alato dà fiato ad una bùccina.

Infine ecco la cascata che s'intaglia fra massi, e nell' apertura si scorge il lontano profilo del castello. Accanto: una faccia mostruosa, la bocca spalancata, come quella dentata della morte e dell'oblio nei poeti antichi. I tratti corrugati, gonfi, sono circonfusi da un alone di alucce di farfalla tempestate di cerchi con un punto in mezzo, segni, la farfalla, di risurrezione, il cerchio puntato, di solarità. La cervice regge una sfera fasciata da bande rilevate, che sembra roteare, punteggiata da quattro rose, sormontata da una torre poggiata sul polo ed assai simile alle casette che venivano poste nelle tombe etrusche. La maschera è quella della morte, del futuro, su cui si leva una sfera, che è la forma del sole, e della mente, dice Macrobio nel Commento al Sogno di Scipione. Il tempo fluisce dal futuro verso il presente, ha il volto della morte divoratrice ma su di essa poggia la mente, al cui vertice sta la torre della saggezza, che trova il punto immobile del moto.

Si torna indietro, ma digradando ancora, accompagnando il corso del ruscello nascosto fra cespugli, fino ad un altro salto delle acque, dove si ergono due statue sbozzate con torva, rustica mano, un gigante che torce le cosce ad una femmina rovesciata con la testa in terra. Il gigante è appoggiato ad un'armatura completa di spada con elsa a borchia, di schinieri, gonna, cimiero a forma di rosa a cinque petali (simbolo del libero arbitrio), di pettorale con la Medusa istoriata sopra. Una lapide mutila parla del colosso di Rodi (dell'isola della Rosa) che sarebbe emulato da quest'altro. Un ciclope che vince un titano, cioè una forza naturale che sottomette l'altra nella vicissitudine eterna su cui l'uomo saggio deve sapersi levare? Nessun passo di mitografo o di poema medievale soccorre. Marsilio Ficino nel De amore ricorda che il Logo divide a metà le anime, e prima di cadere nel corpo gli uomini hanno due facce, onde guardano al materiale ed allo spirituale con pari provvidenza. Ma, piombate nella carne, "è come se fussino nel mezzo divise e delle facce resta una sola per cui ogni volta che volgano la faccia alla bellezza sensibile sono private del- 
l'altra." Il gruppo tozzo e ripugnante ricorda dunque l'orrore primordiale della calata nel corpo, quando il Logo, il gigante, ci rovesciò in basso e spaccò in due, lasciandoci una sola faccia, dopo averci staccati dall'armatura, che nel Pimandro designa il sistema di sfere celesti. Nessuna ferocia nella faccia del gigante, assorto, rivolto altrove.

Si continua a scendere fra alberi che celano con le fronde un gruppo di statue nel punto piú intimamente basso, all'ultimo salto del torrente, che qui risuona scrosciando, e ancora piú doveva un tempo, quando era deviato per due bracci ad avviluppare le pietre scolpite. Una tartaruga grande come un bue, squisita nella fattura minuziosa, reca sulla corazza vellutata dal muschio un vaso rovesciato che regge un globo dove poggia appena il calcagno una figurina la cui veste fa vela al vento; tiene le braccia levate come a suonare un flauto, la testa rovesciata, invisibile.

La tartaruga avanza verso l'acqua su cui protende una testa elegante, e sotto, sull'altra sponda, è spalancata una fauce come di balena dove forse dovrebbe mulinare l'acqua.

Accanto alla tartaruga è una vasca tutta sghemba; al centro si leva un masso su cui pigia gli zoccoli un piccolo Pegaso alato.

Terzo elemento in fila, una macina da mulino, sghemba anch'essa, ma orientata in modo contrario alla vasca. Dirimpetto a questa macina, che serve da sedile, benché inclinata, è scolpito su una pietra un ceppo, le cui radici paiono il grembo biforcuto di Melusina.

Seduti sulla macina del mulino della fortuna, del tempo, si guarda ciò che non è radicato, cioè secondo il libro di Giobbe, ciò che è malvagio: il ceppo sollevato in aria, sorgente di bene se confitto in terra. Volgendo il capo si osserverà un'illustrazione del simbolo: guardando dalla macina sembra d'essere paralleli alla tartaruga e non si è, ma conforta a crederlo, la linea storta della vasca secondo il gioco di falsa prospettiva della sorte, che pone in uno o altro luogo a caso.

La tartaruga, che sta sulla linea dritta, è bestia dalle centinaia di uova, ed è anche, negli "Emblemi" dell'Alciato, simbolo di prudenza e previdenza. Sant'Ambrogio scrisse: "finché vive la testuggine sta nel fango, ma quando muore della sua corrazza si fa uno strumento." E nell'alchimia essa rappresenta la massa confusa, che attraverso il vaso della trasmutazione fiorisce in forme piene e perfette. Ma la figurina in corsa e la lenta tartaruga illustrano il motto. "festina lente" — affrettati con lentezza - che si interpretava nel Rinascimento ermetico come - medita a lungo ed agisci senza riflessione. La figurina è per cadere nelle fauci del cetaceo? Forse a significare, secondo le lezioni di Pico e Ficino, la 
caduta necessaria dell'anima nella vita della materia. (La balena è la bestia dell'Oceano primordiale, su cui sta il dio Oceano, ricorda Boccaccio nella Genealogia deorum gentilium).

La vasca inclinata porta al centro la fonte Elicona, ed il Pégaso spicca il volo alzandosi in direzione obliqua ma contraria alla vasca: "Sic tu Pegaseis si petis aethera pennis / Consilioque animi monstra superba domas" dice un emblema dell'Alciato. Il consilium guida, dice Ficino, la fortuna, adeguandola al fato. Solo salendo sul cavallo pegaseo è dato colpire la Chimera, solo rovesciando le inclinazioni umane.

Dopo questo gruppo il sentiero s'incurva, segue il ruscello dall'alta sponda, e s'inoltra in un amabile ninfeo guardato, all'entrata, da due leoncini emblemi di saggezza. Due fontane sormontate da delfini, animali salvatori provvidenziali come il cavallo pegaseo, stanno a cospetto delle nicchie numerose, ricavate in un muro il cui zoccolo aggetta a formare ricci e anse. Nelle nicchie sono le figure delle tre grazie allacciate, di cui una volta il tergo. Significano che dando amore si riceve bellezza e voluttà, che guardando a Dio si viene cinti da doni, e con la nudità esortano alla franchezza. Scrisse Pico: "si conosce il giusto modo di procedere della teologia orfica sapendo che l'unità di Venere è spiegata nella trinità delle grazie, l'unità della necessità nella trinità del Fato," Ficino in una lettera a Salviati e Benivieni dice che le tre figure rappresentano la necessaria sintesi di animo, corpo e fortuna. Il triplice ritmo insegna l'emanazione dall'uno (la dolorosa inversione, lo squarcio rappresentato dal gruppo del gigante), la conversione per cui si rovescia l'ordine rovesciato, dando senza risparmio, profondendoci e lasciando a Dio ogni cura, negando l'ordine sublunare che è quello del cane infernale, e infine il ritorno all'uno (Pico elenca emanatio, conversio, remeatio). Basta volgersi verso il futuro, sorgente d'ogni vita e bocca di morte, guardare senza alcuna preoccupazione alla fonte d'ogni oscuro pensier (come dice una scritta mutila sulla parete del ninfeo).

Uscendo dal luogo umido, ombroso dominato dal verde e dal turchino, il sentiero piega ed esce su uno spiazzo ampio dove si rizzavano su alte steli Giani bifronti e quadrifonti, simbolo, dice Pico, delle anime capaci di guardare provvedendo alle cose materiali ed alle spirituali, di avere sott'occhio le quattro direzioni della rosa dei venti. Il mondo delle cause seconde, della fortuna ed il mondo delle cause prime, del destino.

Lo spiazzo è solenne, leggiadramente chiuso da un muro, forse un tempo ornato di scale che portavano sul poggio. Nel muro, fra teste in altorilievo di déi dalle corna lunate (forse effigi di Nettuno che, secondo Fulgentius Metaphoralis, ha il corno della ragione e 
quello della libertà con cui si difende dal male), si apre un incavo dove una fanciulla vestita d'una tunica regge una conchiglia da cui spande acqua a riempire la vasca a forma di grifo o drago (forse affiorarono grifi etruschi nel suolo), animale salutare che vola portando l'abbondanza modesta e virginale. O meglio, di Arpia, l'animale di Nettuno, che distrugge col disprezzo i beni terrestri (secondo il Fulgentius Metaphoralis).

Avanzando per la spianata si vede una casa addossata al poggio, che ne emerge col secondo piano. È pendente, di stile piú munito che fiorito. La pendenza dà malessere, anche se si è distratti a guardare un orso che sta contro l'angolo inferiore reggendo lo stemma degli Orsini, a decifrare due cartigli, l'uno che dedica l'edificio al cardinale Mandruzio, l'altro che proclama nello stile delle targhe del castello: Animus quiescendo fit prudentior, ergo. - Darsi requie affinché l'animo cresca in prudenza, - detto sotto una casa sghemba è un invito ironico, poiché di certo una casa promette requie, ma l'inclinazione garantisce inquietudine. È la casa della fortuna, dove non bisogna cercare quiete né rifugio. Se si vorrà trovarvi albergo, entrare in queste due stanze del primo piano, si patirà. La nausea torce lo stomaco appena varcata la soglia, fra le mura storte sul pavimento in salita: si corre alla finestra, si cerca con gli occhi la rocca lontana, e, a vincere la vertigine, il cielo. Il costruttore ha raggiunto il fine: ha insegnato a non dimorare nella casa della fama o della fortuna, e altresí che il solo modo di non essere travolti dal disgusto nel mondo delle cause seconde è di tenere la testa alzata verso il cielo, diventando prudenti. Prudenza, dice Macrobio, "sta nel disprezzare questo mondo e tutto ciò che in esso si trova, in grazia della contemplazione delle cose divine."

La casa ci ha appreso la fuga oppure il dimorare con sguardo fisso fuori della finestra?

Con la casa cessa il cammino, poiché il viottolo finisce in una breve platea con una vasca (si è stati finora nel mondo delle acque inferiori) guardata da sfingi (sfinge è allegoria delle cause del male, ignoranza e fortuna, nell'Alciato). Sul piedestallo d'una di queste sfingi limitari è scritto: - Chi con ciglia inarcate e labbra strette non va per questo loco manco ammira le famose del mondo moli sette. - Saranno le sette meraviglie del mondo antico o le moli, che nel trattato ermetico di Della Riviera del 1602, sono i sette metalli trasmutabili, le sette radici miracolose della natura, di nera radice e di bianco fiore.

$\mathrm{Si}$ piega per un sentiero acclive che gira attorno alla casa, e si giunge alla porta del piano superiore, che emerge sul ciglio, e vi si congiunge con un ponticello agile. Questa parte superiore è viep- 
piú significativa per il camino che aggiunge alla sensazione di familiare conforto, ma il fuoco che salirà per la cappa diritta sarà perpendicolare alla terra, aggraverà la nausea contrastando con le pareti oblique. Il fuoco tende al cielo come lo sguardo di chi sia insofferente del mondo della fortuna. Si è passati dal regno orizzontale delle acque a quello verticale del fuoco; seduti a riposare sul ponticello, osservando la cinta d'alberelli che nasconde una platea di statue, si è sorpresi dal silenzio, il ruscello è lontano.

Fatti pochi passi si è accolti in una spianata teatrale, al cui fondo si staglia stupenda la statua d'un vecchio seduto con un drappeggio sulle gambe, il torso largo, la faccia solenne dalla barba a boccoli folti. Lo circonda uno stagno.

Verso di lui porta la spianata circondata di urne, alla sua destra è un elefante che solleva o atterra un uomo, ha una torre sulla groppa ed un tamburino issato sull'occipite. A destra di chi entra nella platea e fronteggia il vecchio Oceano, sta una figura femminile la cui testa diventa un vaso, sicuramente Teti, la quale sta lontana da Oceano, dice Omero, affinché l'universo non sia soverchiato dalla loro prolificità e infatti la figura è circondata da genietti, uno le sta nella destra, l'altro le scosta i capelli per mormorarle nell'orecchio. Scrive Boccaccio: "Teti senza dubbio è un'acqua, la quale, dice Crisippo, per forza di fervor celeste è tratta dalle viscere della terra," essa è sposa di Oceano "onde pare che marito e moglie sieno la stessa cosa . . . ma Teti è acqua elementata, ovvero che abbia mistura d'altri elementi, per opera della cui mistura può concepire e nutrire." Il vaso sulla testa conferma: è il cratere attraverso il quale lo spirito divino passa nel mondo, secondo il simbolo di Macrobio. Essa sta ad occidente di Oceano perché è uno scadere dell'originaria purezza, per contemplarla bisogna voltare le spalle ad Oceano e la si visita girandole attorno. I putti s'infittiscono sul dorso: due si sporgono nell'arrampicata sú per le spalle, un altro è stato preso dietro le ginocchia da due genii alati con la coda di pesce, ed ora lo rovesciano, come a purificarlo scuotendolo nell'aria, secondo l'antica usanza del "vaglio" o abburrattamento del giovane alle cerimonie di pubertà, a ripetizione del gesto della levatrice, che fa entrare l'aria nel polmoni trasformando il feto in uomo.

Le urne simulano la terra cotta, la mescolanza di terra e fuoco, dove le acque sono quelle superiori, attratte dal fervore celeste. Sono recipienti della verità discepoli di Oceano, secondo il detto alchemico: "Omnia sunt unum in uno circulo sive vase." Quelle che stanno vicino all'Oceano recano due scritte: a sinistra - Fonte non fu tra chi 'n guardia stia delle piú strane belve, - a destra Notte e giorno noi stiam vigili e pronte a guardar da ogni ingiuria 
questa fonte. - Forze belluine respingeranno chi si accosti alla fonte della vita in modo molesto; chi avrà paura delle belve, degli istinti sarà privato d'ogni forza ed alimento. Come dice Giamblico: soltanto la gentile persuasione può conferire con gli istinti, con la generazione. Notte e giorno insieme vincolati in sintesi perfetta costituiscono Dio, affermava la teologia orfica.

Ma chi avanzi dalla mescolanza di divino e materiale di Tetide alla divinità di Oceano, alla prodigalità senza limiti, incontra l'elefante turrito, bardato alla maniera indiana. In India Ganes è Dio dell'intelligenza, e anche l'elefante di piazza della Minerva a Roma, spiega la scritta del suo piedestallo, è effige della mente robusta, capace di sopportare il peso della sapienza. È un malconsigliato chi ha voluto accostarsi alla fonte senza il soccorso dell'elefante, il guerriero che viene piegato dalla proboscide, oppure è colui che ha rinunciato alla sua vita e che l'elefante solleva per deporlo nella torre vuota? La robusta mente solleverà l'uomo che si abbandoni ad essa come sconfitto. Un tamburino suona la pelle tesa dell'animale morto (Sant'Agostino dice che Cristo è teso sulla croce a formare ur tamburo che chiama alla salvezza).

Dietro l'elefante un drago dalla coda scagliosa, le ali spiegate, stritola un leoncino o molosso e alza le zampe a colpire altri due leoni o molossi ringhianti; ha le ali cosparse di mezzelune e di fiamme, come a dire la sua natura di creatura ignea ma sublunare, soggetta alla crescita e alla diminuzione della luna. L'ermetismo raccomandava di lasciare gli istinti alla loro agonia, poiché naturam natura vincit; ma sarebbe conforme a teologia se il drago fosse la disarmonia lunare delle passioni viziose che lottano peraltro con la superbia solare, rappresentata dai cani.

$\mathrm{Da}$ quel combattimento sempre rinnovato l'uomo può trarre beneficio, poiché equilibrandosi i due movimenti del cuore egli sarà libero. Si lascino da parte i due tremendi avvertimenti, e ci si inoltri dietro gli animali in lotta: appare una bocca mostruosa addossata ad un clivo. Sulle labbra era forse scritto Lasciate ogni pensier voi ch'entrate; entrando, si scopre una stanza nella roccia, dove piove luce dall'alto dai due occhi. Un sedile corre attorno il muro, un trapezio di pietra è la tavola dove si dovrebbe consumare il sacrificio, forse quello dei ricordi, degli oscuri pensier di cui è padre il futuro.

A sinistra dell'Oceano si scendono alcune scalette, accanto a delle fauci (della balena su cui sta Oceano?), ci si inoltra in un dedalo verde dove affiora d'un tratto una donna riversa addormentata fra l'erba. L'anima che sogna, invece di migliorare? Infatti, poco dopo ci si ritrova in vista dei giganti, delle figure del mondo delle acque infere. Occorre tornare indietro se non si vuole smar- 
rire ciò che si è acquistato, sicché si passa ancora a cospetto di Oceano, si rivede l'elefante. I suoi paramenti indiani suggeriscono che i costruttori sapessero che il Viçuddha Chakra è un centro dell'anima simboleggiato da un elefante bianco, e denota il momento della liberazione in cui si domina il tempo nella sua triplicità: passato, presente, e futuro.

I mostri che stanno attorno: la bocca divoratrice, il drago che in eterno colpisce con le zampe i leoni che in eterno lo azzannano, sono ben rappresentanti della sorte o fortuna, del caso, che Proclo definisce come la forza demoniaca capace di legare cause fra loro staccate, destini fra loro distinti.

Un candelabro si alza solitario, raccogliendo in sé ciò che sarebbe abbandonato a se stesso, sparso e disperso, casuale. Poi s'incontra una belva accosciata, pacifica, di fattura cinese, e una nicchia con un sedile al suo riparo; vi è scritto: - Voi che pel mondo gite errando vaghi di veder meraviglie alte e stupende, venite qua dove son facce orrende elefanti leoni orsi orchi et draghi. -

A oriente comincia la rampa di una scalinata ampia di pietra, e salendola ci si ritrova alla platea delle ghiande e delle pigne, simboli che ci aiutano a sventare gli inganni del demone che intreccia i vincoli apparenti e mostruosi della fortuna, abituando a fermare l'attenzione sui vincoli necessari e giusti del destino.

Infine si incontra di nuovo Cerbero e la sagoma lieve del tempietto, sapendo ora quale naturale preghiera sia da levare nella sua cella ottagonale.

Università di Roma 